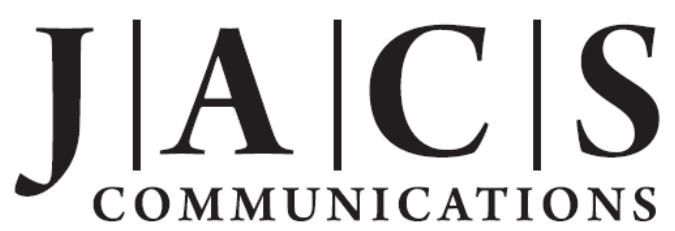

\title{
Heterogeneous Catalysis of a Copper-Coated Atomic Force Microscopy Tip for Direct-Write Click Chemistry
}

Walter F. Paxton, Jason M. Spruell, J. Fraser Stoddart*

Department of Chemistry, Northwestern University, 2145 Sheridan Road, Evanston, Illinois 60208

E-mail: stoddart@northwestern.edu

\section{Supporting Information}

Microcontact Printing of Alkyne
Molecules onto Azide-Terminated
Silicon Wafer Pieces. Our procedure
for preparing surfaces containing
microcontact printed features for
comparison to features written via
direct-write click chemistry (adapted
from Ref. S1) was as follows: Polydimethylsiloxane (PDMS) stamps were prepared from a 10:1 mixture of PDMS-Sylgard Elastomer 184 and Sylgard Curing Agent 184 (Dow Corning Corp. Midland, MI), poured over a fluoro-coated patterned silicon wafer - SAM of (tridecafluoro-1,1,2,2tetrahydroocty)-1-trichlorosilane (Gelest, Inc., Morrisville, PA) - in a Petri dish. The mixture was allowed to stand at room temperature for $1 \mathrm{~h}$ before being placed into an oven at $60{ }^{\circ} \mathrm{C}$ for $16 \mathrm{~h}$. The cured PDMS was then peeled away from the patterned silicon wafer and cut into $\sim 1 \mathrm{~cm}^{2}$ pieces and used as stamps. These stamps were oxidized in UV/ozone plasma (200 W, $10 \mathrm{sec}$ ) and stored in deionized $\mathrm{H}_{2} \mathrm{O}$ until ready for use. Oxidized PDMS stamps were removed from $\mathrm{H}_{2} \mathrm{O}$ and dried under a stream of $\mathrm{N}_{2}$, and were then immediately inked with 1 drop of 1 $\mathrm{mM} \mathrm{CuSO}{ }_{4} \cdot 5 \mathrm{H}_{2} \mathrm{O}$ in EtOH, 1 drop of 2 $\mathrm{mM}$ ascorbic acid in $\mathrm{EtOH}$, and 4 drops of $5 \mathrm{mM}$ terminal alkyne in $\mathrm{EtOH}$. This mixture was allowed to combine on the

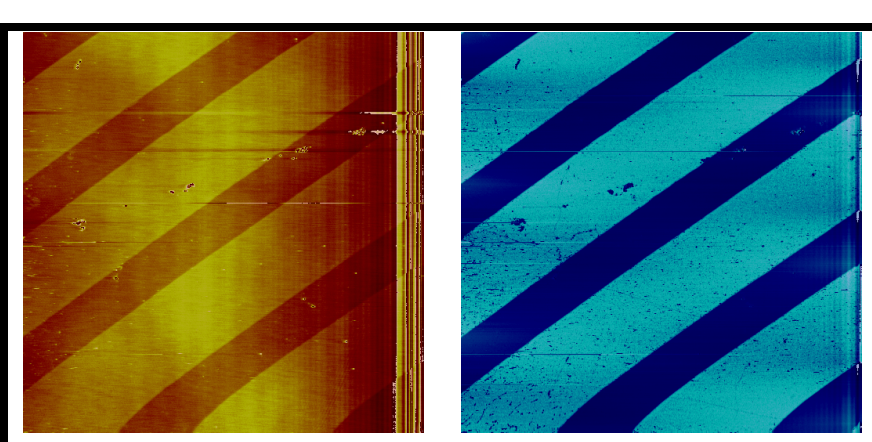

Figure S1 - $50 \times 50$ micron region contact mode atomic force microscopy topography (left) and friction (right) images in $\mathrm{EtOH}$ of 4-pentynoic acid monolayers stamped onto azide-terminated silicon wafers. Topography image indicates a height of 1.0(2) nm. Friction image indicates the carboxylic acid terminated regions exhibit higher friction than the unreacted (azide-terminated) regions.

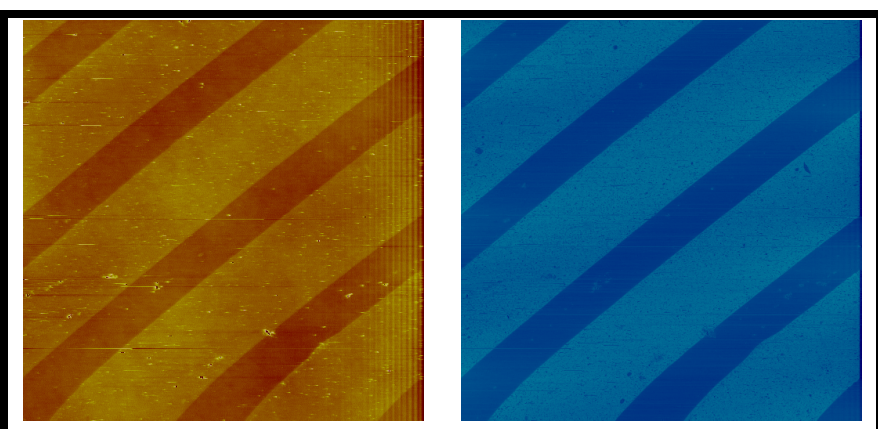

Figure S2 - $50 \times 50$ micron region contact mode atomic force microscopy topography (left) and friction (right) images in $\mathrm{EtOH}$ of 2,5,8,11-tetraoxatetradec13-yne (TEG-alkyne) monolayers stamped onto azideterminated silicon wafers. Topography image indicates a height of $1.0(1) \mathrm{nm}$. Friction image indicates the TEG-alkyne-terminated regions exhibit higher friction than the unreacted (azide-terminated) regions. 
stamp for 30 sec before being removed from the stamp under a stream of $\mathrm{N}_{2}$. The stamp was placed immediately onto an azide-terminated silicon wafer piece for at least $1 \mathrm{~h}$. The stamp was then removed from the silicon wafer piece which was rinsed liberally in $\mathrm{EtOH}$, followed by deionized $\mathrm{H}_{2} \mathrm{O}$, and then dried under a stream of $\mathrm{N}_{2}$ and stored in a fluoroware container, protected from heat and light. Figures S1-S3 show AFM images (topography and friction) of typical features prepared by microcontact printing the

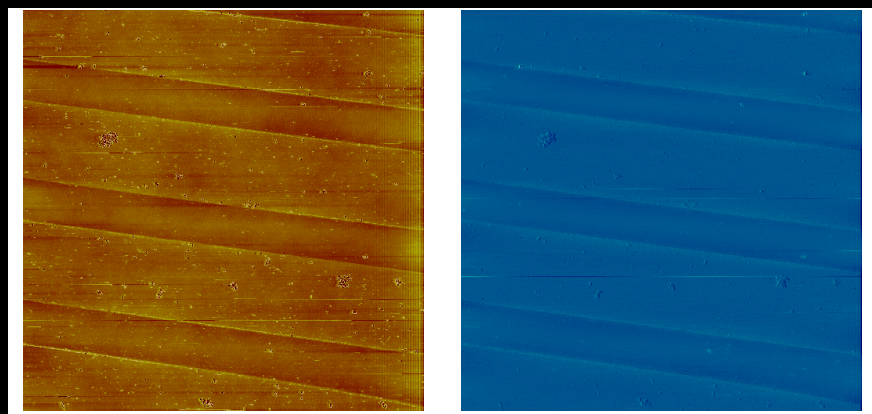

Figure S3 - $50 \times 50$ micron region contact mode atomic force microscopy topography (left) and friction (right) images in $\mathrm{EtOH}$ of propargylamine monolayers stamped onto azide-terminated silicon wafers.

three compounds used in this communication: 4-pentynoic acid, propargylamine, and 2,5,8,11-tetraoxatetradec-13-yne (TEG-alkyne). Analysis of microcontact printed films by contact mode AFM indicated that the carboxylic acid terminated regions were approximately $1 \mathrm{~nm}$ taller than unreacted azide-terminated regions.

Direct-Write Click Chemistry of Propargylamine and TEG-Alkyne. Writing of alkynes onto azide surfaces was also possible with propargylamine and TEG-alkyne (Figures S4-S5) under the same conditions used to write 4-pentynoic acid.
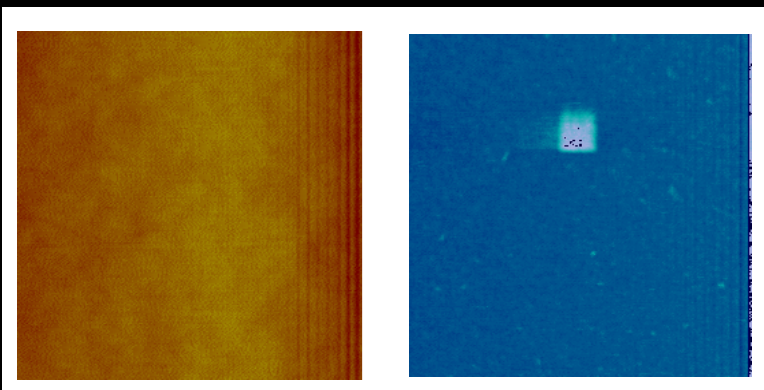

Figure S4 - Contact mode AFM images in an ethanolic solution of propargylamine $(50 \mathrm{mM})$ using a copper-coated AFM tip. Topography (left) and tip-substrate friction (right) of a $10 \times 10 \mu \mathrm{m}$ region at low force $(30 \mathrm{nN})$ and high speed (40 $\mu \mathrm{m} / \mathrm{s}$ ) after writing a $1 \times 1 \mu \mathrm{m}$ square (center) at high force $(280 \mathrm{nN})$ and low speed $(2 \mu \mathrm{m} / \mathrm{s})$.

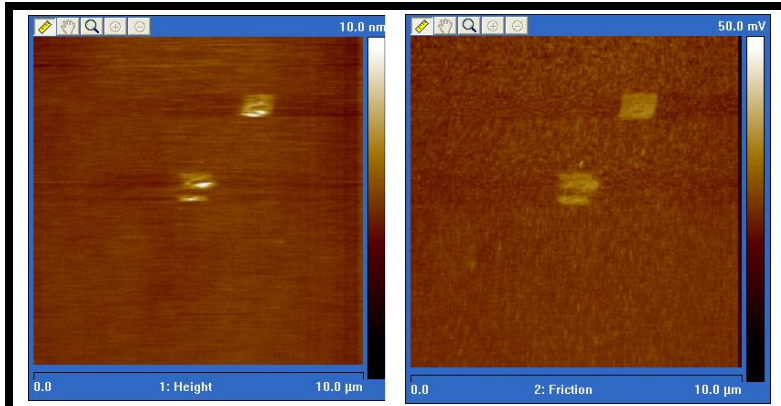

Figure S5 - Contact mode AFM images in an ethanolic solution of 2,5,8,11-tetra-oxatetradec13-yne (50 mM) using a copper-coated AFM tip. Topography (left) and tip-substrate friction (right) of a $10 \times 10 \mu \mathrm{m}$ region at low force (30 $\mathrm{nN})$ and high speed $(40 \mu \mathrm{m} / \mathrm{s})$ after writing features at high force $(350 \mathrm{nN})$ and low speed $(2 \mu \mathrm{m} / \mathrm{s})$. 
Control Experiments. Attempts to reproduce direct-write click chemistry using SiN AFM tips on azide-terminated surfaces in $50 \mathrm{mM}$ 4-pentynoic acid in $\mathrm{EtOH}$ failed. A constant scan rate of 2 $\mu \mathrm{m} / \mathrm{s}$ for writing at forces $<400 \mathrm{nN}$ failed to produce any raised features of higher friction. Writing with a force $\sim 400 \mathrm{nN}$ appeared to perturb the monolayer, possibly because of monolayer destruction. ${ }^{\text {S2 }}$ Control experiments involving a copper AFM tip and $50 \mathrm{mM}$ of ethanolic alkyne (4-pentynoic acid) on an azide-terminated surface produced similar results, confirming that both alkyne and a copper AFM tip are required to pattern an azide surface using this method. The contact mode topography and friction images shown in Figure $\mathbf{S 6}$ - taken after attempting to write TEG-alkyne onto an azide surfaces using an unmodified SiN AFM tip - are representative of the results of these control experiments.

Materials. All reagents were obtained from commercial sources and were used as received. For AFM experiments we used $100 \% \mathrm{EtOH}$ (Chromasolv, Sigma-Aldrich) and silicon-nitride AFM tips (DNP-S; Veeco Instruments, Woodbury, NY) with nominal tip radii of $10 \mathrm{~nm}$ and force constants ranging from $0.5-1 \mathrm{~N} / \mathrm{m} .2,5,8,11$-Tetraoxatetradec13 -yne was prepared using a previously described procedure. ${ }^{33}$

AFM. All AFM work was performed on a Bioscope II (Veeco Instruments, Woodbury, NY) at the NUANCE Center at Northwestern University. All AFM imaging was done in contact mode. All images were subject to standard image processing protocols, including image flattening and contrast enhancement.

\section{REFERENCES}

(S1) Spruell, J. M.; Sheriff, B. A.; Rozkiewicz, D. I.; Dichtel, W. R.; Rohde, R. D.; Reinhoudt, D. N.; Stoddart, J. F.; Heath, J. R. Angew. Chem. Int. Ed. 2008, 47, 9927-9932.

(S2) Onclin, S.; Ravoo, B. J.; Reinhoudt, D. N. Angew. Chem. Int. Ed. 2005, 44, 6282-6304.

(S3) Eder, E.; Preishuber-Pflugl, P.; Stelzer, F. J. Mol. Catal. A: Chem. 2000, 160, 6369. 